\title{
Créativité et traduction spécialisée
}

\author{
Élisabeth Lavault-Olléon
}

\section{(Q) OpenEdition}

Journals

Édition électronique

URL : http://journals.openedition.org/asp/3460

DOI : $10.4000 /$ asp.3460

ISSN : 2108-6354

\section{Éditeur}

Groupe d'étude et de recherche en anglais de spécialité

\section{Édition imprimée}

Date de publication : 1 décembre 1996

Pagination : 121-133

ISSN : 1246-8185

\section{Référence électronique}

Élisabeth Lavault-Olléon, « Créativité et traduction spécialisée », ASp [En ligne], 11-14 | 1996, mis en ligne le 29 avril 2013, consulté le 19 avril 2019. URL : http://journals.openedition.org/asp/3460 ; DOI : 10.4000/asp.3460

Ce document a été généré automatiquement le 19 avril 2019.

Tous droits réservés 


\title{
Créativité et traduction spécialisée
}

\author{
Élisabeth Lavault-Olléon
}

1 Pour beaucoup, la traduction se confond avec le thème et la version (que chacun a pratiqués à un moment ou un autre de sa scolarité), ou avec la traduction littéraire (où l'on « oublie » trop souvent le traducteur). Or, si l'on considère la traduction d'un point de vue professionnel, ce sont là des applications soit artificielles soit marginales de la traduction.

2 La traduction - non littéraire et non pédagogique - est omniprésente dans notre vie quotidienne, depuis le guide d'utilisateur du dernier logiciel acheté jusqu'au plus récent ouvrage sur la culture des orchidées, en passant par le western du mardi soir. Si on l'oublie, c'est que dans la plupart des cas, elle a atteint son objectif, transmettre de façon transparente ce qui aurait été incompréhensible dans la langue d'origine, à tel point que le texte semble avoir été conçu dans la langue d'arrivée.

3 Cette traduction-là, qui recouvre $90 \%$ du volume de traduction mondial, n'a les faveurs ni des linguistes, ni du grand public : sous l'étiquette large de "traduction technique », elle est assimilée à un travail répétitif et non créatif, à une traduction sans intérêt et sans liberté. De plus, littéraires et linguistes y voient un exercice bâtard car il semble nécessiter des connaissances techniques approfondies, et de ce fait rester réservé à ceux qui sont parfois davantage des spécialistes d'un ou de plusieurs domaines spécialisés que des spécialistes de langues.

4 Pourtant le traducteur de textes non littéraires dispose souvent d'une marge de liberté insoupçonnée, qui lui permet d'exercer sa créativité. C'est ce que je voudrais démontrer dans cet article. Les exemples que je citerai proviennent de textes appartenant à des genres et des domaines de spécialité divers, englobés par J. Delisle sous l'appellation de textes «pragmatiques» (1980), regroupant aussi bien des articles scientifiques et des textes de la presse générale ou spécialisée que des textes dits utilitaires ou opératoires issus des entreprises industrielles et commerciales, qui constituent la grande masse des traductions proposées sur le marché aujourd'hui. 


\section{Définir la créativité en traduction}

5 Que recouvre la notion de créativité en traduction? En premier lieu, le pouvoir d'inventer ses propres solutions en traduction, des solutions qui ne sont ni répertoriées dans des outils lexicographiques ni prétablies par des manuels, des solutions que le traducteur fait naître de sa propre interprétation du document à traduire.

6 Les recherches sur la traduction portent plus souvent sur la problématique de la fidélité que sur les aspects créatifs du travail du traducteur. L'aptitude à inventer des solutions inédites est, de plus, peu développée en traduction pédagogique. Les cours de thème et de version prennent souvent comme référence La stylistique comparée du français et de l'anglais (Vinay \& Darbelnet 1958). Les sept procédés de traduction que ses auteurs ont rendus classiques sont abondamment repris et illustrés dans les manuels de version qui se présentent parfois comme des catalogues de recettes plus ou moins stéréotypées.

7 Vinay et Darbelnet ont cependant bien averti au départ qu'ils n'offraient pas "une collection de recettes» mais que par «une confrontation des deux stylistiques, la française et l'anglaise ", ils souhaitaient « dégager les lignes générales et même des lignes précises» (1958: 26-27) qui caractérisent les génies respectifs des deux langues en présence. Ces lignes générales sous-jacentes à tout le livre, ces « traits caractérologiques du français et de l'anglais » ont été regroupés par Jean Delisle à la fin de son premier ouvrage (1980 : 244, annexe III). Si les traducteurs chevronnés ont oublié les fameux procédés (aux contours bien arbitraires), ils sont certainement imprégnés des idées reprises par ce tableau, qui souligne, entre autres, la préférence de l'anglais pour le concret, le pragmatique, la juxtaposition, l'ordre chronologique des événements, et celle du français pour l'articulation, l'interprétation, la nominalisation, etc.

La connaissance profonde des deux stylistiques, autant issue de l'étude qu'acquise par l'expérience, permet au traducteur de mettre en œuvre des automatismes et de traduire de façon quasiment réflexe en s'appuyant alors sur la mise en correspondance systématique des éléments de deux langues. Mais cette activité réflexe, si fondamentale qu'elle soit en traduction, n'est qu'une partie de l'activité traduisante. En fait, les limites de la stylistique comparée sont énoncées par ses propres auteurs lorsqu'ils disent vouloir « observer le fonctionnement d'une langue à une autre » (id.), ce qui les place sur le plan de l'analyse de la langue et non du discours. L'analyse contrastive, qu'elle soit approfondie dans une optique pédagogique à partir de corpus d'erreurs (Ballard 1987) ou dans une optique linguistique à la lumière de la théorie des opérations énonciatives (Chuquet \& Paillard 1987) ne manque pas de laisser le traducteur professionnel non littéraire insatisfait, car elle se réfère prioritairement à l'exercice de version littéraire et s'appuie sur la résolution de problèmes de langues qu'il a le plus souvent résolus dans sa pratique.

9 Il est frappant de constater que tous les ouvrages relevant de cette approche parlent essentiellement de traduction et pratiquement jamais du traducteur. De ce fait, la créativité en traduction n'est pas mentionnée.

La théorie qui a, pour beaucoup, relayé l'analyse contrastive, à savoir la théorie interprétative de la traduction (Seleskovitch \& Lederer 1984) se concentre, quant à elle, non pas sur la comparaison d'énoncés traduits, mais sur le processus de traduction luimême. Elle accorde une place prépondérante aux composantes extra-linguistiques du 
discours et refuse une approche linguistique de la traduction comme transfert « d'une langue à une autre " voire "d'un texte à un autre». Elle donne au traducteur un rôle moteur dans l'activité traduisante car c'est lui qui interprète le texte en fonction de ces paramètres extra-linguistiques, qui en restitue le sens et fait pour cela les choix de reformulation qu'ils imposent.

Toute traduction comporte certes des correspondances entre des termes et des vocables, mais elle ne devient texte que grâce à la création d'équivalences. C'est là l'élément central de notre théorie. (Lederer 1994 : 55)

11 La théorie interprétative de la traduction, source de frustration pour beaucoup parce qu'elle évacue systématiquement tous les problèmes de traduction qui relèvent seulement de la langue, a le mérite d'être unifiante, en ce qu'elle énonce des principes suffisamment généraux pour qu'ils s'appliquent à tout couple de langue et à quasiment tout type de texte. Son principe est l'intervention du traducteur dans l'opération traduisante, non pas comme machine à reproduire des correspondances mais comme être « interprétant » qui fonctionne à l'aide de son bagage cognitif et affectif et par sa prise de conscience d'une méthode fondée sur l'interprétation. Son corollaire est la reconnaissance de la créativité du traducteur quel que soit son domaine d'intervention :

Du style le plus neutre au style le plus recherché, il ne saurait y avoir de traduction de textes sans création d'équivalences, dérivées de l'action des connaissances non linguistiques sur les significations de la langue. (Lederer 1994 : 41)

On pourrait arguer que créativité et création d'équivalences ne sont pas synonymes, que la première implique un pouvoir d'invention qui construit à partir du néant - la page blanche de l'écrivain - alors que la création d'équivalences que pratique le traducteur s'exerce à partir d'un matériau existant, le texte source.

Le talent créateur du traducteur ne se manifeste pas comme celui de l'écrivain par l'expression d'une subjectivité dans le discours esthétique. Il prend plutôt la forme d'une sensibilité exacerbée au sens du texte de départ et d'une grande aptitude à réexprimer ce sens dans un autre texte cohérent et de même force expressive. Pour ce faire, le traducteur dispose d'une liberté relative quant au choix des moyens linguistiques. L'adéquation d'une pensée et d'une forme exige souvent de lui qu'il fasse preuve de créativité dans l'exploitation des ressources que lui offre la langue d'arrivée. (Delisle 1993 : 151)

13 Pour ma part, je parlerai de créativité chaque fois qu'il y a écart par rapport à des solutions préétablies relevant de la seule prise en compte des correspondances linguistiques (ou stylistiques). Mais cette créativité trouve ses limites dans la fidélité au sens tel qu'il est défini par la théorie interprétative (Lavault, 1985: 50), à savoir « exprimer le vouloir-dire de l'auteur avec l'effet voulu en respectant les contraintes de la langue cible ».

\section{Contraintes et libertés dans les textes pragmatiques}

Dans la traduction professionnelle, les paramètres extralinguistiques ont une importance cruciale :

- Nature et fonction de la traduction, à quel genre appartient le texte et quelle est sa «mission »?

- Qualité du donneur d'ordre (est-ce une entreprise qui doit promouvoir une certaine image de marque, un intellectuel connu pour ses prises de position polémiques, un chercheur qui cherche la reconnaissance de ses pairs en s'inscrivant dans le macrotexte de sa spécialité ?) 
- Cible de la traduction (initiés, grand public, utilisateur...) ils entraînent le plus souvent une plus grande liberté par rapport au linguistique, et de ce fait une plus grande marge de créativité pour le traducteur. Selon C. Durieux, «le traducteur doit épouser la mission du texte » (1991 : 171). À partir du moment où il est établi, par exemple, que la mission d'un texte technico-commercial est de convaincre l'acheteur potentiel, le traducteur dispose d'une relative liberté dans le choix de ses formulations.

Citons l'exemple de Christine Durieux (1991: 172) sur les modes d'emploi et trois façons de formuler une commande des plus banales :

Formulation neutre - Pour exécuter la fonction X, appuyez sur la touche A puis sur la touche B.

Formulation positive (promotionnelle) - Pour exécuter la fonction X, il suffit d'appuyer sur la touche A puis sur la touche B.

Formulation négative (critique) - Pour exécuter la fonction $\mathrm{X}$, il faut non seulement appuyer sur la touche A, mais encore sur la touche B.

17 Dans la querelle des «sourciers" et des "ciblistes" - les partisans de la fidélité prioritaire au texte source contre les partisans de la fidélité prioritaire au public cible les traducteurs de textes pragmatiques se situent plutôt du côté cibliste, contrairement aux traducteurs littéraires qui se sentent plus liés au texte source.

La traduction nécessite toujours une adaptation complète du document d'origine à un public qui se caractérise par des habitudes différentes, des goûts différents, des modes de pensée différents, des comportements différents. Un public, donc, qui devra recevoir le document traduit comme si ce dernier avait été rédigé par quelqu'un de même culture. (Gouadec 1989 : 3)

Cette visée cibliste de la traduction de textes pragmatiques montre bien où se situent les contraintes et les libertés du traducteur spécialisé. Ce ne sont pas celles du traducteur littéraire.

Envisageons maintenant la créativité du traducteur sous quatre angles : la terminologie, la création discursive, la réécriture induite par les déficiences du texte source et l'adaptation.

\section{La créativité en terminologie}

Elle est l'expression la plus évidente de la créativité du linguiste puisqu'il faut bien créer un terme pour dénommer une réalité nouvelle, abstraite ou concrète. Dans la majorité des traductions techniques, le traitement de la terminologie prend un temps considérable, mais il s'appuie souvent sur des documents fournis et validés par le client ou établis à la suite d'une recherche documentaire.

Plus le texte est utilitaire, plus le traducteur a des chances de travailler avec une terminologie préétablie. Mais plus la technologie présentée est inédite, plus il se trouve devant des lacunes terminologiques. Même s'il est recommandé au traducteur de ne pas prendre de risques en ce domaine et de laisser aux terminologues et aux experts du domaine le soin de créer la terminologie requise, quitte à leur en laisser la paternité en ne traduisant pas le terme source, il arrive souvent que le donneur d'ordre attende du traducteur qu'il propose une solution inédite. 

traduction. Il y a pléthore de produits nouveaux, il y a profusion de termes angloaméricains liés à ces produits. Malgré leurs efforts, les commissions de terminologie restent souvent à la traîne, les produits nouveaux arrivent sur le marché, suivis de près par la traduction de leur documentation. Le plus souvent, ce sont les traducteurs, employés par les entreprises ou les sociétés de traduction chargés de la localisation des produits, qui créent la terminologie.

Je citerai pour exemple le cas d'une de nos stagiaires de DESS qui se retrouve chargée par l'entreprise de traduire un glossaire regroupant tous les termes-clés d'un produit informatique qui arrive actuellement sur le marché français. Ce produit, connu jusqu’à présent des spécialistes français sous son appellation américaine générique de « workflow ", recouvre une nouvelle solution technique pour l'automatisation des travaux administratifs. La société française en question, qui va être la première à commercialiser un produit de ce type a demandé au traducteur de ne pas utiliser le terme « workflow » car elle veut justement se démarquer de ses concurrents américains. Dans un cas comme celui-ci, le travail de création se fait heureusement en équipe avec les ingénieurs qui travaillent sur le produit, mais il ne se limite pas à trouver une appellation pour un seul concept, il implique forcément la création d'autres termes dérivés du premier.

Même si les produits existent depuis quelque temps, la terminologie dans le domaine des nouvelles technologies de l'information est en fait encore très floue et très fluctuante. C'est ce que nous avons constaté lors de la traduction collective d'un ouvrage sur les réseaux, et en particulier sur le logiciel Netscape (Kent 1996). Quel terme adopter, par exemple, pour les fameux logiciels d'accès au réseau Internet que bon nombre d'utilisateurs français appellent toujours «browsers »? Dans le cas de la traduction d'un ouvrage non lié à un logiciel précis, la traductrice a choisi le terme "butineur ", qui est dans l'air depuis quelques mois, aux dépens de "browser", "logiciel d'accès", " navigateur », et même " fureteur ». Il n'y a pas eu création, mais un choix conscient qui, si l'ouvrage de vulgarisation se vend bien, aidera à la fixation d'une traduction originale et évocatrice.

\section{Les créations discursives}

Elles interviennent à chaque fois que la traduction par correspondances n'est pas possible, ou ne produit pas l'effet escompté sur le public récepteur et n'est alors pas fidèle au sens. Le traducteur doit alors puiser dans l'ensemble de ses connaissances et de ses expériences passées et explorer toutes les possibilités d'expression de la langue cible pour trouver une formulation, qui, comme le fait remarquer Delisle, n'est jamais consignée dans un dictionnaire puisque l'équivalence ainsi créée, qualifiée de ponctuelle, est propre au contexte qui l'a rendue possible. Cette création d'équivalences inédites existe au niveau lexical, comme le montrent les deux exemples suivants :

Exemple 1 (tiré d'un article scientifique sur la flore des Alpes australiennes, Revue de géographie alpine, 1992) :

A number of exotic weed species have spread throughout the Alps creating costly and difficult control and eradication problems. Blackberry (Rubus fruticosus) is a good example, this species having been spread through part of the Alps by Baron Ferdinand von Mueller during his scientific surveys... 
On aurait pu penser qu'un terme aussi simple que « exotic » pouvait être traduit par le terme correspondant "exotique». Mais la ronce commune peut-elle être comprise comme plante « exotique " par un lecteur français, même lorsque ce terme apparaît dans un texte sur l'Australie? Le traducteur, tenant compte de sa cible et des connotations tropicales de ce terme, déplacées dans ce contexte, a créé une équivalence inédite ; il a également précisé qu'il s'agit bien des Alpes australiennes :

Un certain nombre d'espèces végétales non indigènes a proliféré dans les Alpes australiennes, créant des besoins coûteux de contrôle et de destruction de ces espèces. La ronce commune en est une...

Exemple 2 (texte de vulgarisation scientifique portant sur les dangers supposés des téléphones portables) :

No one really understands the long-term health consequences of holding a microwave transmitter next to your brain because nobody has thoroughly studied them.

Personne ne connaît encore les effets à long terme que peut avoir sur la santé le fait de tenir un émetteur de micro-ondes à proximité du cerveau puisqu'aucune étude sérieuse n'a encore été faite sur le sujet.

La traduction de "microwave transmitter» par «émetteur de micro-ondes" est linguistiquement et scientifiquement correcte. Pourtant, elle produit un effet fâcheux du fait de l'adéquation en français d'aujourd'hui du terme initialement technique « microondes » à " four à micro-ondes », qui produit une série d'associations d'idées porteuses de malentendus sur le sujet de l'article. Le traducteur a donc dû rechercher un terme équivalent non connoté dans la langue générale et, en l'occurrence, mettre en œuvre, outre son intuition, ses connaissances scientifiques pour trouver un équivalent (Lavault, 1994 : 46-48):

Personne ne connaît encore les effets à long terme que peut avoir sur la santé le fait de tenir un émetteur d'hyperfréquences à proximité du cerveau puisqu'aucune étude sérieuse n'a encore été faite sur le sujet.

Les créations discursives s'exercent aussi sur des parties de phrase, voire sur des phrases entières. Ainsi dans un ouvrage sur le multimédia (Rathbone 1994), un tableau (voir tableau 1) présentait les recommandations du spécialiste pour le type d'achat à effectuer, en fonction du « fiddling level » de l'utilisateur, traduit ici par « niveau de bricolage ».

Tableau 1. Recommandations du spécialiste

\begin{tabular}{|l|l|}
\hline $\begin{array}{l}\text { Your level of } \\
\text { fiddling }\end{array}$ & verdict \\
\hline $\begin{array}{l}\text { I love fiddling } \\
\text { with } \\
\text { computer my }\end{array}$ & $\begin{array}{l}\text { Buy the current rage in multimedia parts and install them all yourself. } \\
\text { Beware, however: the latest, greatest technology almost always has } \\
\text { problems that haven't had time to work themselves out. }\end{array}$ \\
\hline $\begin{array}{l}\text { I like to fiddle } \\
\text { with } \\
\text { computer my }\end{array}$ & $\begin{array}{l}\text { Buy the parts separately and install them yourself. Buy parts in the middle } \\
\text { price range and avoid the cheapest stuff. }\end{array}$ \\
\hline I fiddle a little bit & $\begin{array}{l}\text { Buy an upgrade kit. Because these parts already get along, the installation is } \\
\text { a lot easier. }\end{array}$ \\
\hline
\end{tabular}


Get that thing away from me!
Buy a new computer with everything already installed. Sure, it's hard saying good-bye to the old one, but keep reminding yourself of all those free compact discs that come with a new computer.

\section{Rathbone 1994}

La traduction des deux phrases en caractères gras ne peut être ni littérale ni résolue par les dictionnaires ou la seule connaissance de la langue. Elle donne lieu à des créations discursives qui naissent de la connaissance du contexte et du style de l'ouvrage, ainsi que du bagage cognitif personnel du traducteur.

Pour la quatrième catégorie, la catégorie 0 représentée par « Get that thing away from me! », le traducteur, ayant bien compris la situation de ce " non bricoleur ", pouvait produire une série de paraphrases en langue cible : " je suis nul en bricolage », "je déteste bricoler ", " je n'y connais rien », " je ne veux pas y toucher », etc. En effet à partir du moment où il est clair que pour réexprimer le sens plus lisiblement, on se démarque des formulations de la langue source, les options peuvent se multiplier. Le choix final s'opère selon des critères personnels du traducteur ; ici le respect du ton humoristique et provocateur renforcé par la tournure exclamative, et la prise en compte de l'effet de rupture après la liste précédente (I love, I like, I fiddle...), l'a amené à préférer « je n'ai même pas de tournevis ! ( (voir tableau 2).

Tableau 2. Traduction des recommandations

\begin{tabular}{|l|l|}
\hline $\begin{array}{l}\text { Votre niveau de } \\
\text { bricolage }\end{array}$ & Suggestion \\
\hline $\begin{array}{l}\text { J'adore bricoler } \\
\text { mon ordinateur }\end{array}$ & $\begin{array}{l}\text { Achetez les modules et les périphériques les plus sophistiqués et installez-les } \\
\text { vous-même. Attention toutefois, les technologies les plus nouvelles et les } \\
\text { plus géniales s'accompagnent souvent de problèmes qui n'ont pas encore } \\
\text { été décelés ni résolus. }\end{array}$ \\
\hline $\begin{array}{l}\text { J'aime bien } \\
\text { bricoler mon } \\
\text { ordinateur }\end{array}$ & $\begin{array}{l}\text { Achetez les modules séparément et installez-les vous-même. Choisissez des } \\
\text { produits milieu de gamme et évitez les produits trop bon marché. }\end{array}$ \\
\hline $\begin{array}{l}\text { Je bricole un } \\
\text { tout petit peu }\end{array}$ & $\begin{array}{l}\text { Achetez un kit d'extension multimédia. Comme les composants sont prévus } \\
\text { pour fonctionner ensemble, l'installation est beaucoup plus facile. }\end{array}$ \\
\hline $\begin{array}{l}\text { Je n'ai même } \\
\text { pas } \\
\text { tournevis ! de }\end{array}$ & $\begin{array}{l}\text { Achetez un nouvel ordinateur avec les modules tout installés. Bien sûr, c'est } \\
\text { un peu triste de se séparer de sa vieille machine, mais pensez à tous les CD- } \\
\text { RoMs gratuits qui sont fournis avec tout ordinateur multimédia! }\end{array}$ \\
\hline
\end{tabular}

Le livre sur la toute dernière version de Netscape mentionné plus haut (Kent 1996) est un ouvrage technique qui présente peu de difficultés de traduction, une fois réglés les problèmes terminologiques. Par exemple, voici la description d'une des boîtes de dialogue du logiciel :

You have the three following choices: 
Save to Disk. Click here to see the Save As dialog box, in which you can tell Netscape where to put the file once it has transferred it.

Cancel transfer. Click here to remove the dialog box and return to the document. Configure a Viewer. Click here to tell Netscape which program to send the file to.

Traduction :

Trois possibilités s'offrent à vous :

Enregistrer sur le disque dur. Cliquez sur ce bouton pour afficher la boîte de dialogue Enregistrer Sous, dans laquelle vous dites à Netscape où placer le fichier une fois qu'il l'a transféré.

Annuler transfert. Cliquez sur ce bouton pour supprimer la boîte de dialogue et revenir au document.

Configurer un afficheur. Cliquez sur ce bouton pour dire à Netscape sur quel programme il doit envoyer le fichier.

Pourtant, la première phrase du premier chapitre est la suivante :

Everyone and his dog seem to be using Netscape these days.

Et un peu plus loin dans cette même première page, on trouve :

Right now, Netscape is still on top (though it's being given a real run for its money by Internet Explorer, Microsoft's Windows 95 browser).

Pour ces expressions idiomatiques, toutes les deux métaphoriques et appartenant au registre familier, il n'existe pas de correspondance préétablie et le traducteur se trouve d'emblée devant deux cas où il doit produire une équivalence inédite.

Dans le premier cas, il est plus que probable qu'il choisira par commodité de perdre l'image (qui est purement gratuite) et de traduire par «tout le monde semble utiliser Netscape aujourd'hui », sans chercher à créer de paraphrases qui rendraient davantage le ton familier et joueur :

- Qui (donc) n'utilise pas Netscape aujourd'hui ?

- Y a-t-il quelqu'un qui n'utilise pas Netscape aujourd'hui ?

- Tout le monde, même Madame Michut, se sert de Netscape aujourd'hui, etc.

Mais pour la deuxième phrase, la création discursive est indispensable :

- Aujourd'hui, Netscape est toujours numéro un,

- Bien que la concurrence de Microsoft Explorer, le navigateur Microsoft sous Windows, lui donne du fil à retordre

- Bien qu'il soit dangereusement concurrencé par Microsoft Explorer

- Même si la concurrence de Microsoft Explorer le met sérieusement dans l'embarras

- Bien qu'il ait à subir les attaques de Microsoft Explorer, etc.

Ces exemples montrent que les créations discursives sont également nécessaires dans des textes techniques. Il est vrai qu'ils proviennent d'un texte technique qui s'adresse au grand public. Dans les documents techniques au sens strict, qui sont produits par et pour l'industrie (les nomenclatures, les notices de montage rédigées par et pour des techniciens etc.), la fonction prioritairement descriptive et informative des textes prête souvent plus à la traduction par correspondances préétablies qu'à la création d'équivalences. Certains de ces textes supportent d'ailleurs assez bien la traduction automatique. Mais on constate que, dès qu'un document technique fait l'objet d'une diffusion commerciale, il est écrit en grande partie dans une langue courante, accessible et agréable au lecteur. En d'autres termes, pour arriver à vendre du texte technique, il faut l'enrober, et c'est cet enrobage qui est le plus retors à la traduction. C'est cet enrobage qui sollicite la créativité du traducteur. 
, le texte technique a une autre caractéristique qui met à l'épreuve la créativité du traducteur. Il est souvent mal rédigé ou ambigu, et doit parfois être entièrement réécrit par le traducteur.

\section{La créativité induite par les déficiences du texte source}

39 Je parlerai ici de deux cas de figure que j'ai souvent rencontrés. Premièrement, il est extrêmement fréquent que le texte source soit mal écrit, souvent parce qu'il a été rédigé par un technicien qui connaît bien le produit mais n'est pas un spécialiste de la rédaction. La langue est parfois incorrecte ou ambiguë et surtout, le texte présente un manque de logique et de cohérence au sein des paragraphes et d'un paragraphe à l'autre. Il a visiblement été écrit commande par commande, ou étape par étape, et donc souvent phrase par phrase. Aucun travail global n'a été fait sur le texte.

e deuxième cas est typique des articles scientifiques, en sciences dures comme en sciences humaines. Ces articles sont très souvent écrits en anglais par des chercheurs qui ne sont pas anglophones. Ils présentent des incorrections et des maladresses qui en obscurcissent le sens et ils demandent au traducteur un énorme effort de compréhension, voire d'interprétation, car il faut parfois passer par la langue maternelle de l'auteur (quand on la connaît) pour retrouver le sens en décelant les interférences typiques entre cette dernière et l'anglais.

41 Compte tenu des contraintes «ciblistes » que j'ai mentionnées plus haut, à savoir l'adaptation à la mission du texte et au public cible, le traducteur ne peut pas se contenter d'une traduction incertaine ou maladroite sous prétexte de fidélité au texte source. Dans ces deux cas, le traducteur doit réécrire le texte, lui redonner une homogénéité sur le plan lexical, reconstruire une logique en remodelant la syntaxe, et veiller à la cohérence globale, en redécoupant les phrases et les paragraphes. Il s'agit bien ici d'un travail d'écriture qui est intrinsèquement créatif.

\section{L'adaptation}

42 Le besoin d'adaptation culturelle est flagrant dans tous les textes commerciaux ou publicitaires, et dans ces domaines, le traducteur joue un rôle créatif essentiel pour la réussite du produit. Dans le genre journalistique, où règnent dans les titres les allusions, les références et les citations culturelles plus ou moins tronquées, le traducteur peut amplement satisfaire son génie créatif. Je citerai l'exemple du titre d'un article dont j'ai déjà parlé et qui portait sur les dangers potentiels des téléphones portables : «Dial P for Panic ».

Il y a là une allusion au film d'Hitchcock Dial $M$ for Murder, dont l'adaptation française est Le crime était presque parfait. Le traducteur doit alors proposer une équivalence qui présente elle aussi une allusion, si possible au cinéma ou à une situation courante, par exemple: Psychose, Psychose au téléphone, Panique au téléphone, Un coup de fil peut coûter une vie.

Cet exemple rejoint la traduction pédagogique, où la traduction des titres est un exercice relativement classique. Mais ce goût de la formule se retrouve dans les ouvrages de 
vulgarisation technique et scientifique. Dans l'ouvrage déjà cité sur le multimédia (Rathbone 1994), on trouve le titre de chapitre suivant, qui traite de l'utilisation du multimédia sous Windows : "Making Windows scream and flash », qui a été traduit par une équivalence inédite et culturellement marquée: «Les spectacles son et lumière sous Windows ".

Quand, dans le même ouvrage technique, on explique longuement comment s'y prendre pour enlever les taches de "peanut butter» sur un CD-ROM, l'adaptation culturelle s'impose.

Pour conclure, un autre type d'adaptation en traduction spécialisée peut être décrit comme une " adaptation discursive ", c'est-à-dire une adaptation au genre, ou plutôt au type de document qui est à produire dans la langue cible en fonction de la culture cible. Divers travaux sur l'analyse des discours spécialisés montrent, par exemple, qu'une brochure touristique ou une notice pharmaceutique ne sont pas rédigées et présentées de la même façon en France, aux États-Unis et en Grande-Bretagne. Une étude sur la presse médicale française et anglo-saxonne fait apparaitre que les médecins français se montrent plus catégoriques, plus prescriptifs, voire moralisateurs, alors que leurs homologues britanniques mettent davantage en avant les données scientifiques (Régent 1994 : 58). Cette disparité reflète certainement les différences socioculturelles profondes qui existent entre le corps médical des deux côtés de la Manche. Le traducteur, quant à lui, peut, en se fondant sur de telles études, effectuer une adaptation qui portera sur la rhétorique du discours, pour que sa traduction soit conforme au style de cette famille discursive. Les modifications peuvent être subtiles, limitées à une atténuation ou un renforcement de la modalité et à l'usage plus ou moins grand des «hedges» ou précautions oratoires (Dudley-Evans 1994). J'ai cité plus haut des exemples de traductions d'ouvrages informatiques caractérisés par le style à la fois familier et ludique qui est propre aux informaticiens américains. La question d'une adaptation dans un registre moins familier se pose constamment pour le traducteur (cf. plus haut «everyone and his dog...») et peut donner lieu, me semble-t-il, à un travail créatif, puisqu'il s'écarte délibérément d'une traduction qui ne prendrait pas en compte ce contexte socioculturel.

47 J'espère avoir montré, par ces exemples, que la créativité du traducteur est sollicitée, même dans des textes de spécialité. Comment s'exerce-t-elle, quels sont les processus cognitifs qui permettent au traducteur de réécrire le sens avec ses propres formulations? Les trois étapes de la théorie interprétative, interprétation du sens, déverbalisation et reformulation sont mises en œuvre. Mais c'est surtout dans la troisième phase que la compétence créative ou associative du traducteur s'exerce. Elle implique l'exploration systématique des ressources expressives de la langue cible, et donc une connaissance profonde de celle-ci, et elle s'appuie sur un raisonnement qui fonctionne par analogie avec des expressions existantes. Elle nécessite aussi une bonne dose de sensibilité et d'intuition qui nourrissent une imagination "créatrice» ou plutôt une imagination «recréatrice» (Delisle 1993: 152) indispensable à tout traducteur et pour l'instant heureusement encore absente des machines. 


\section{BIBLIOGRAPHIE}

Ballard, M. 1987. La traduction : de l'anglais au français. Paris : Nathan Université.

Chuquet, H. et M. Paillard. 1987. Approche linguistique des problèmes de traduction. Paris : Ophrys.

Dudley-Evans, T. 1994. « Academic Text: The Importance of the Use and Comprehension of

Hedges ». ASp 5-6, 1994, 131-140.

Durieux, C. 1990. "Liberté et créativité en traduction technique ». La Liberté en Traduction, Actes

du Colloque international. Paris : Didier Érudition, 169-179.

Delisle, J. 1980. L'analyse du discours comme méthode de traduction : initiation à la traduction française de textes pragmatiques anglais. Ottawa : Éditions de l'Université d'Ottawa.

Delisle, J. 1993. La traduction raisonnée. Ottawa : Presses de l'Université d'Ottawa.

Gouadec, D. 1989. Le traducteur, la traduction et l'entreprise. Paris : AFNOR.

Kent, P. 1996. Using Netscape 2 for Windows 95. Indianapolis : Que Corporation (Traduction en cours).

Lavault, E. 1985. Fonctions de la traduction en didactique des langues. Paris : Didier Érudition.

Lavault, E. 1994. « Du téléphone à micro-ondes au cheval de Troie : quelle culture pour le traducteur spécialisé ?». ASp 5-6, 45-54.

Lederer, M. 1994. La traduction aujourd'hui. Paris : Hachette F.L.E.

Rathbone, A. 1994. Multimedia and CD-ROMs for dummies. San Mateo : IDG Books Worlwide Inc. (traduit par J-M. Herellier et C. Wolosin, Multimedia et CD-ROM pour les nuls, Paris : Sybex, 1994).

Régent, O. 1994. «L'article scientifique : un produit culturel ». ASp 5-6, 55-60.

Seleskovitch, D. et M. Lederer. 1984. Interpréter pour traduire. Paris : Didier Érudition.

Vinay, J-P. et J. Darbelnet. 1958. Stylistique comparée du français et de l'anglais. Paris : Didier.

\section{RÉSUMÉS}

À partir de plusieurs exemples tirés de traductions spécialisées, notamment dans le domaine de l'informatique, l'auteur soutient que le traducteur technique fait preuve de créativité, que ce soit par la création de terminologie, par la création discursive, par la réécriture de textes défectueux dans la langue source et par l'adaptation.

On the basis of translation examples from specialised fields and especially information technology, the author claims that creativity is needed by the technical translator who very often has to coin new terminology, resort to cultural adaptation as well as to the creation of discourse equivalences, and rewrite faulty or incomplete source text. 
INDEX

Mots-clés : adaptation, créativité, théorie interprétative, traducteur, traduction

Keywords : adaptation, creativity, interpretive theory, translation, translator

\section{AUTEUR}

\section{ÉLISABETH LAVAULT-OLLÉON}

Elisabeth Lavault-Olléon est maître de conférences à l'université Stendhal Grenoble 3.

elisabeth.lavault@u-grenoble3.fr 